\title{
Comprehensive Longitudinal Analysis of the Five Sisters Hot Springs in Yellowstone National Park Reveals a Dynamic Thermoalkaline Environment
}

\section{Jesse T Peach}

Montana State University Bozeman https://orcid.org/0000-0003-4095-5242

Rebecca C Mueller

Montana State University

Dana J Skorupa

Montana State University

Margaux M Mesle

Montana State University

\section{Sutton Kanta}

Montana State University Bozeman

\section{Eric Boltinghouse}

Montana State University Bozeman

\section{Bailey Sharon}

Montana State University Bozeman

Valerie Copiè

Montana State University Bozeman

\section{Brian Bothner}

Montana State University

Brent M Peyton ( $\sim$ bpeyton@montana.edu )

Montana State University

\section{Research Article}

Keywords: Thermoalkaline, archaea, bacteria, hot springs, mass spectrometry, small molecule characterization

Posted Date: June 15th, 2021

DOl: https://doi.org/10.21203/rs.3.rs-603209/v1

License: (c) (i) This work is licensed under a Creative Commons Attribution 4.0 International License. Read Full License 


\section{Abstract}

Research focused on microbial populations of thermoalkaline springs has been driven in a large part by the lure of discovering functional enzymes with industrial applications in high-pH and high temperature environments. However, the fundamental ecology of these springs has largely been overlooked. To better understand the functional outcomes of interactions between the geochemistry and the microbial community of thermoalkaline springs, we conducted a three-year study of the Five Sisters (FS) springs that included high-resolution geochemical measurements, $16 \mathrm{~S}$ rRNA sequencing of the bacterial and archaeal community, and mass spectrometry based extracellular and intracellular small molecule characterization. By combining all four datasets, we completed a comprehensive analysis of the intricate thermoalkaline spring system. Over the course of the study, the microbial population responded to changing environmental conditions, with archaeal populations decreasing in both relative abundance and diversity when compared to bacterial populations. Decreases in the relative abundance of Archaea were associated with environmental changes that included decreased availability of specific nitrogen and sulfur containing extracellular small molecules. The multi-factorial analysis suggests a complex and dynamic environment with an elastic microbial community that responded to geochemical and extracellular small molecule transitions.

\section{Introduction}

Thermoalkaline springs are unique environments of biological and industrial significance. Commercial applications are well documented in these systems and current thermoalkaline bioprospecting efforts are broad [1]. A successful example is the commercialization of a suite of thermostable enzymes including lipolytic and hydrolytic enzymes [2,3]. Of particular interest is the development of thermostable cellulolytic enzymes capable of converting lignocellulosic biomass into sugars and ultimately ethanol under industrial conditions [4]. The potential to develop thermo- and pH-stable enzymes for commercial applications is driving research in this area, with a lesser focus on the fundamental ecology of these systems $[5,6]$.

As bioprospecting has driven an increase in the number of described thermoalkaline springs, new microbial lineages and metabolic networks have been uncovered [2]. However, despite increased interest and investigation in these systems, gaps in knowledge remain. For example, the temporal dynamics of thermoalkaline microbial populations across multiple years have rarely been investigated [7] and to our knowledge, analyses of the small molecule compositions have not been conducted. This is especially true of winter sampling of hot springs in YNP where there is limited access. Gaining ecology-based understanding of thermoalkaline environments has broad implications including improving culturing efforts.

Although relatively rare, thermoalkaline springs can be found in several locations around the world, including Yellowstone National Park (YNP) where they are prevalent $[1,8,9]$. One such group of springs in YNP includes the Five Sisters (FS) hot springs, located in the White Creek Drainage (WCD). WCD is part of 
Lower Geyser Basin, the largest thermal basin in YNP. Thermal features in WCD have been previously identified as areas likely to contain distinct and dynamic environments $[10,11]$. In this study, the FS thermoalkaline springs were examined over the course of three years and a comprehensive analysis was conducted using high-resolution geochemical measurements, 16S rRNA microbial community sequencing, and liquid chromatography mass spectrometry (LCMS) based small molecule characterization that enabled the establishment of temporal microbial trends and a better understanding of driving factors underlying shifts in microbial population makeup and metabolism in this unique environment.

\section{Methods}

\section{Description of the Five Sisters Site}

The FS site (RCN Database LWCG023A, LWCG023B, LWCG023C) consists of a group of alkaline-chloride springs located in the south-east corner of the Lower Geyser Basin in the WCD $\left(44.5325^{\circ} \mathrm{N}, 110.7971^{\circ} \mathrm{W}\right)$ (Figure 1A). White Creek flows down the drainage and is accompanied by several thermoalkaline springs including Spindle Geyser (LWCG149) and two of the best studied sites in YNP, Octopus Spring (LWCG138) and Mushroom Spring [12]. The FS site is located a few meters south of White Creek against a steeply inclined hill and consists of three springs and five distinct pools, labeled 1 to 5 from East to West, with variable interconnectivity (Figure 1B). FS1 is fed by a small geyser and is connected above-ground to FS2 and FS3. There is no visible above-ground connection between FS3 and FS4, although there may be below-ground connectivity between the two springs. FS4 and FS5 are connected above-ground and FS5 outflow continues away from the spring group and feeds into White Creek. FS1 is the largest spring at several meters wide and deep, followed by FS5, FS3, FS2 and FS4.

\section{Sample collection}

Samples were collected within one week of the first day of March in 2017, 2018 and 2019. Stainless steel cups on extendable poles ( $0.25 \mathrm{~L}$ total volume) were used to collect samples from each spring. The cups were rinsed with spring water first and then used to collect a small amount of sediment slurry. Samples were collected in triplicate and were taken at three distantly located locations within each spring with paired subsamples for metabarcoding and mass spec analysis collected at each sampling location. Sediment slurry samples were composed of $\sim 8 \mathrm{~mL}$ of sediment with $\sim 7 \mathrm{~mL}$ of thermal water and were placed in sterile $15 \mathrm{~mL}$ conical centrifuge tubes after collection (Corning, Corning, NY). Slurry samples were immediately frozen in a dry ice and ethanol bath in the field and stored on dry ice until transported to Montana State University (MSU) and placed in a $-80^{\circ} \mathrm{C}$ freezer until genomic or mass spectrometry analysis.

\section{Geochemical analysis}

Aqueous geochemistry was monitored with each sampling event. Briefly, the temperature and $\mathrm{pH}$ of each site was measured in situ using a combined pH-temperature probe (Hach HQ30d, Hach Co., Loveland, 
CO). Dissolved oxygen was measured in the field using the High Range Dissolved Oxygen method and a portable colorimeter (Hach DR900, Hach Co., Loveland, CO). Total dissolved metals were analyzed using $0.22 \mu \mathrm{m}$ filter-sterilized water acidified with $5 \%$ trace metal-grade nitric acid. Concentrations of total metals were quantified using an Agilent $7500 \mathrm{ce}$ ICP-MS by comparing to certified standards (Agilent Technologies, Environmental Calibration Standard 5183-4688) at MSU's Environmental and Biofilm Mass Spectrometry Facility. Samples for anion analysis were filtered through $0.22 \mu \mathrm{m}$ filters, and the filtrate was analyzed using a Dionex ICS-1100 chromatography System (Dionex Corp., Sunnyvale, CA) equipped with a $25 \mu \mathrm{L}$ injection loop and an AS22-4×250 mm anion exchange column, using an eluent concentration of $4.5 \mathrm{mmol} \cdot \mathrm{L}^{-1}$ sodium carbonate and $1.4 \mathrm{mmol} \cdot \mathrm{L}^{-1}$ sodium bicarbonate flowing at a rate of $1.2 \mathrm{~mL} \cdot \mathrm{min}^{-1}$. Samples for total carbon (TC), total nitrogen (TN), non-purgeable organic carbon (NPOC), and dissolved inorganic carbon (IC) were filtered through $0.22 \mu \mathrm{m}$ filters, and the filtrate deposited in ashed glass vials filled with no headspace and capped with a septum. A Shimadzu TOC-CSH instrument with an attached TN module (Shimadzu Scientific Instruments, Columbia, MD) was used to measure TC/TN/NPOC/IC at MSU's Environmental Analytical Lab (EAL). Filtered samples acidified with sulfuric acid (final $\mathrm{pH}<2$ ) were also sent to the EAL for ammonium analysis using a Lachat QuickChem 8500 flow injection analyzer (Hach Co., Loveland, CO).

\section{DNA extraction and metabarcoding analysis}

DNA was extracted from each replicate using the FastDNA ${ }^{\mathrm{TM}}$ Spin Kit for Soil kit (MP Biomedicals) according to the manufacturer's instructions, with an additional 40 s bead beating step. The $\mathrm{V} 4$ region of the 16S rRNA gene was targeted using the latest versions of the 515F-80R primers [13], 515F-A (GTGYCAGCMGCCGCGGTAA; [14]) and 806R-B (GGACTACNVGGGTWTCTAAT; [15]) using Phusion Hot Start II Hi Fidelity polymerase in 25 ul reactions. Thermocycling conditions were an initial denaturation at $98^{\circ} \mathrm{C}$ for $30 \mathrm{~s}, 22$ cycles of $98^{\circ} \mathrm{C}$ denaturation for $15 \mathrm{~s}$, annealing at $58^{\circ} \mathrm{C}$ for $30 \mathrm{~s}$, extension at $72^{\circ} \mathrm{C}$ for $20 \mathrm{~s}$, with a final extension at $72^{\circ} \mathrm{C}$ for 5 minutes. To facilitate multiplexing, dual-index barcodes were added in a second PCR using the Nextera kit (Illumina Inc.) with 10 cycles as above, but with an annealing temperature of $55^{\circ} \mathrm{C}$. PCR reactions were quantified using the Quant-It HS dsDNA kit (Invitrogen) and measured using a Biotek $\mathrm{H} 2$ plate reader. Reactions were pooled at equal concentrations and sequenced on a MiSeq using V3 600 cycle kits.

Paired-end reads were merged, primers were removed, and sequences were quality filtered with an expected error rate of 0.5 using USEARCH $[16,17]$. Operational taxonomic units (OTUs) were identified using UNOISE3, which identifies biological sequences clustered into zero-radius OTUs (ZOTUs), similar to amplicon sequence variants. ZOTU tables were generated by mapping reads back to representative ZOTU sequences. Taxonomy of ZOTUs was identified using the IDTAXA online classifier via DECIPHER (http://DECIPHER.codes) [18]. Archaeal sequences were added to a reference tree generated using full and near full-length $16 \mathrm{~S}$ sequences downloaded from GenBank and the Genome Taxonomy database [19]. Sequences were aligned using mafft and the reference phylogeny was constructed using maximum 
likelihood with RAxML[20,21]. Environmental sequences were aligned to the reference and added to the tree using pplacer, and the combined trees were annotated using the Interactive Tree of Life [22,23].

\section{Sediment small molecule extraction}

Sediment samples were extracted using several methods resulting in small molecule fractions that were characterized by LCMS analysis. Extraction began by thawing frozen samples in a $40^{\circ} \mathrm{C}$ water bath and dislodging microbes from the sediment layer. Microbes were dislodged through two one-minute rounds of agitation on a vortex machine. Well-mixed samples were then centrifuged for five-minutes at 400 RPM to create a sediment pellet. The clear supernatant was then collected and placed in a clean vial and the procedure was repeated with the addition of a $5 \mathrm{~mL}$ Milli-Q water wash. Wash supernatant was added to the original sediment-free supernatant. Combined supernatant was then centrifuged at 10,000 RPM for 15 minutes to create a cell pellet. The supernatant was collected in a clean vial for extracellular solidphase extraction (SPE) analysis and enough phosphate buffer solution (1X PBS) was added to the cell pellet to completely cover the pellet.

The extracellular layer was first acidified to a pH of 2 using formic acid (Fisher Chemical, Hampton, ND) and Agilent Bond Elut PPL solid phase extraction cartridges (Agilent Technologies, Santa Clara, CA) were prepared. Preparation involved two cartridge volume additions of methanol (Fisher Chemical, Hampton, $\mathrm{NH}$ ) followed by two cartridge volume additions of Milli-Q water and a final cartridge volume addition of methanol. SPE cartridges were then connected to a SPE manifold (VacMaster 10, Biotage, Uppsala, Sweden) and a vacuum pump to selectively concentrate and isolate extracellular small molecules. Acidified samples were passed through the cartridges and then placed under nitrogen to dryness. Clean vials were placed below the cartridges and captured extracellular small molecules were eluted using $1 \mathrm{~mL}$ of methanol. Extracellular small molecules were further concentrated under negative pressure using a Concentrator Plus (Eppendorf, Hamburg, Germany) until dry and then stored in a $-80^{\circ} \mathrm{C}$ freezer until ready for LCMS analysis.

The vial containing the cell pellet was centrifuged at 400 RPM and the PBS supernatant was removed. Two cell pellet volumes of extraction buffer were added consisting of $8 \mathrm{M}$ urea (Fisher Chemical, Hampton, NH), 0.1M Tris-HCL (MilliporeSigma, Munich, Germany), 50mM ethylenediaminetetraacetic acid (EDTA) (MilliporeSigma, Munich, Germany) and 1X protease inhibitor mix (MilliporeSigma, Munich, Germany). Cell pellets were next lysed in a two-part procedure. First, cell pellets in extraction buffer were placed in liquid nitrogen for 10 seconds then removed and allowed to thaw. This procedure was repeated three times. After the freeze/thaw procedure, cell pellets underwent two rounds of sonication using a Biologics Inc. Ultrasonic Homogenizer 3000 (Bioloics, Manassas, VA) set at a 40\% duty cycle for three minutes.

After cell lysis, the intracellular samples were centrifuged at 15,000 RPM for 30 minutes to pellet cell debris. The resulting supernatant was removed and placed in a clean vial while the debris were washed with the addition of $50 \mu \mathrm{L}$ of extraction buffer. After agitating the debris with a vortex machine, samples were centrifuged at 15,000 for 30 minutes. The second supernatant was again removed and added to the 
vial containing the first supernatant. Four sample volumes of ice-cold acetone (Fisher Chemical, Hampton, $\mathrm{NH}$ ) was then added to precipitate protein. Samples were placed in a $-80^{\circ} \mathrm{C}$ for two hours to aid precipitate formation. After two hours, samples were centrifuged at 5,000 RPM for five minutes and the intracellular supernatant was collected and placed in a clean vial. As with the extracellular small molecule layer, the intracellular small molecule layer was concentrated under negative pressure to dryness and stored in a $-80^{\circ} \mathrm{C}$ freezer until LCMS analysis. When ready for LCMS analysis, both the intracellular and extracellular small molecule samples were reconstituted with $50 \mu \mathrm{L}$ of methanol:water (50:50) and placed in clean MS vials.

\section{$\underline{\text { LCMS analysis }}$}

Samples were analyzed on an Agilent 6538 Q-TOF MS paired with an Agilent 1290 ultra-high performance liquid chromatography (UHPLC) (Agilent Technologies, Santa Clara, CA) using a $132 \AA$, $2.2 \mu \mathrm{m}, 2.1 \mathrm{~mm} \times 150 \mathrm{~mm}$ Cogent Diamond Hydride HPLC column (Microsolv, Greater Wilmington, NC) located in the Proteomics, Metabolomics and Mass Spectrometry facility at MSU. Ionization was accomplished via electrospray ionization in positive mode. Mobile phases A and B consisted of water with $0.1 \%$ formic acid and acetonitrile with $0.1 \%$ formic acid, respectively. A 15 -minute UHPLC run time was used, starting with $100 \%$ mobile phase B and moving to $30 \%$ B in a linear gradient over 14 minutes. At 14 minutes, mobile phase B was increased back to $100 \%$ for the final minute of the UHPLC run. Flow was kept at $600 \mu \mathrm{L} /$ minute and the column compartment temperature was constant at $30^{\circ} \mathrm{C}$.

LCMS raw data files were converted to .mzML files using MSConvert with Vendor peak picking and data mining was completed using mzMine [24,25]. A minimum intensity of 1,000 counts was used throughout the mining process along with a ppm error of 20 and a time discrepancy of 0.1 minutes for determining unique peaks. Molecules formulas were determined using mzMine's formula generation identification feature with an error of $15 \mathrm{ppm}$. After data mining, blank samples were used to remove residual features from the datasets. Features were only kept in the experimental data if they had an area five times greater than the blank sample. Using this method, almost 5,000 combined features were found in the extracellular small molecule samples and over 4,500 features were found in the combined intracellular small molecule samples. Datasets were then grouped and analyzed using MetaboAnalyst [26]. Samples were removed if not in over $50 \%$ of the samples and the data were filtered using their interquartile range value and normalized using the autoscale function.

\section{Correlation analysis}

The geochemical, 16S rRNA, intracellular and extracellular small molecule datasets were compared using correlograms. This was accomplished by utilizing Bioconductor and mixOmics packages in $\mathrm{R}[27,28]$. Two datasets were compared at a time and the top discriminating features were determined using a partial least-squares discriminating analysis (PLSDA). The top features from each dataset were then compared between springs and years to determine their correlative relationship. An ANOVA was also conducted for each relationship to determine the significance of the correlation. 
Table 1. Geochemical characteristics from 2017-2019

\begin{tabular}{|c|c|c|c|c|c|c|c|c|c|c|c|c|c|c|c|}
\hline \multirow{2}{*}{$\begin{array}{l}\text { Sampling date } \\
\text { Site }\end{array}$} & \multicolumn{5}{|c|}{ February 27, 2017} & \multicolumn{5}{|c|}{ Merch 1, 2018} & \multicolumn{5}{|c|}{ Merch 7, 2019} \\
\hline & FS1 & FS2 & FS3 & FS4 & FS5 & FS1 & FS2 & FS3 & FS4 & FS5 & FS1 & $\mathrm{FS} 2$ & FS3 & FS4 & FS5 \\
\hline SWE & \multicolumn{5}{|c|}{$88 \%$} & \multicolumn{5}{|c|}{$110 \%$} & \multicolumn{5}{|c|}{$124 \%$} \\
\hline Temp (C) & 78 & 78 & 75 & 77 & 66 & 71 & 74 & 73 & 71 & 64 & 75.1 & 80.3 & 79.8 & 80 & 70 \\
\hline $\mathrm{pH}$ & 9 & 8 & 8 & 8 & 8 & 9 & 8.5 & 8.5 & 8.5 & 8.5 & 8.8 & 8.4 & 8.4 & 8.4 & 8.5 \\
\hline $\mathrm{DO}(\mathrm{mg} / \mathrm{L})$ & 1.7 & 1.3 & 1.2 & 1.3 & 1.5 & 0.2 & 0.2 & 0.7 & 0.2 & 0.8 & 2.2 & 0.4 & 0.5 & 0.5 & 4.4 \\
\hline Chloride (mg/L) & 216 & 224.6 & 234.3 & 243.1 & 246.9 & 253.4 & 256.2 & 263.4 & 255.1 & 256.5 & 277.1 & 296.1 & 277.2 & 272 & 291.1 \\
\hline Sulfate (mg/L) & 12.4 & 12.8 & 13.3 & 13.6 & 14 & 14.3 & 14.6 & 14.7 & 14.8 & 15.2 & 11.7 & 11.3 & 11.5 & 11.4 & 12 \\
\hline Sodium (mg/L) & 158.8 & 143.3 & 144.2 & 157.4 & 157 & 140.8 & 152.4 & 141.7 & 161.2 & 158.1 & 169.3 & 170.1 & 173.6 & 170 & 168.2 \\
\hline Potassium (mg/L) & 6.9 & 6.6 & 6.9 & 6.7 & 6.7 & 6.4 & 6.8 & 6.2 & 7.1 & 7.2 & 6.3 & 6 & 7.2 & 7.2 & 7.4 \\
\hline Zine (ug/L) & 9.5 & 12.1 & 11.4 & 11.5 & 12 & 22.4 & 18.4 & 19.2 & 21.9 & 14.6 & 19 & 22 & 20 & 16 & 18 \\
\hline Arsenic (ug/L) & 750 & 670 & 677 & 731 & 715 & 698 & 740 & 667 & 771 & 771 & 800 & 790 & 770 & 780 & 770 \\
\hline Molybdenum (ug/L) & 11.7 & 11.8 & 12.3 & 11.8 & 11.8 & 11.7 & 12.4 & 11.2 & 13 & 13 & 12 & 13 & 12 & 12 & 13 \\
\hline $\mathrm{TC}(\mathrm{mg} / \mathrm{L})$ & 45.3 & 55.3 & 55.2 & 55.3 & 55.7 & 44.7 & 54.5 & 54.1 & 54.8 & 54.4 & 45.7 & 57 & 56.6 & 56 & 55.4 \\
\hline TN (ppm) & 0.20 & 0.08 & 0.08 & 0.09 & 0.09 & 0.05 & 0.06 & 0.06 & 0.05 & 0.05 & 0.13 & 0.1 & 0.1 & 0.1 & 0.1 \\
\hline Inorganic Carbon (mg/L) & 43.9 & 54.4 & 55.2 & 54.4 & 55.5 & 45.3 & 55 & 52.4 & 529 & 54.9 & 44.1 & 53.6 & 53.7 & 54.3 & 53.7 \\
\hline NPOC (mg/L) & 1.1 & 0.3 & 0.2 & 0.4 & 0.3 & 0.4 & 0.5 & 0.3 & 0.5 & 0.3 & 0.8 & 0.6 & 0.6 & 0.6 & 0.5 \\
\hline NH4 (ug N/L) & 25 & 23.1 & 21.5 & 23.6 & 27.8 & 14 & 29 & 20 & 24 & 17 & 32 & 26 & 26 & 24 & 17 \\
\hline
\end{tabular}

SWE denotes snow water equivalent percentage of the median for the previous year. DO is

dissolved oxygen. TC is total carbon. TN is total nitrogen. NPOC is non-purgeable organic carbon.

\section{Results}

\section{Temporal 16S rRNA profiles}

To generate new knowledge on the ecology of thermoalkaline springs, we conducted an extensive analysis which included geochemical, 16S rRNA and small molecule data. We began by investigating the microbial community, focusing on populations that showed variation across the temporal analysis. This was accomplished by exploring the 16S rRNA ZOTU relative abundance data using MicrobiomeAnalyst to determine year and spring specific microbial trends [29]. This analysis revealed several microbial patterns including two striking population trends (Figure 2A). First, a consistent decrease was observed in the relative abundance of microbial ZOTUs from superphylum TACK from 2017 to 2018 and from 2018 to 2019. The second was an increase in relative abundance of ZOTUs from the bacterial phyla Proteobacteria and Deinococcota. Over the course of the study, FS1 and FS5 had a marked increase in Deinococcota relative abundance while FS2, FS3 and FS4 had relative Proteobacteria population increases in 2019 (Figure 2B). Other trends, such as a relative decrease in Acidobacteriota populations in FS3, FS4 and FS5 from 2017 to 2019, were also apparent. Along with a decrease in abundance, archaeal alpha diversity decreased for each spring between 2017 and 2019 (Figure 3). The exception to this pattern was FS5, which displayed an increase in alpha diversity in 2019. The 16S rRNA analysis indicated 
a diverse and elastic microbial community, along with a steady decline in the archaeal diversity from 2017 to 2019.

\section{Temporal geochemistry of the FS site}

The geochemical and small molecule datasets were then examined to determine possible drivers of the temporal archaeal population decreases. We began by exploring the geochemical data and an initial analysis demonstrated how consistent each spring was over the course of the study (Table 1). Temperature and $\mathrm{pH}$, as well as other commonly collected variables like total carbon, showed little change. Analysis via a 2-dimensional principal component analysis (2D-PCA) scores plot, highlighted the trend of similar geochemistry in 2017, 2018 and 2019 (Figure 4A). Annual geochemical fluctuations appeared to be moderate although four geochemical measurements including nitrogen, sodium, sulfate and zinc concentrations, were significantly different between the three years, with ANOVA p-values $<0.05$ (Figure 4B). However, the patterns of concentration changes between 2017 and 2019 for these variables was not linear. Total nitrogen concentrations decreased in 2018 and then increased back to 2017 levels in 2019 (Figure 4B). Sodium and zinc both increased in 2019 relative to 2017 but remained constant between 2017 and 2018 for sodium and 2018 and 2019 for zinc. The pattern of sulfate was also different, increasing in 2018 and then decreasing in 2019 to below 2017 levels. Although not included in the PCA or ANOVA analysis, Table 1 also indicates that snowpack, which ultimately turns into spring runoff, was variable between years. Snow water equivalents (SWE) in 2016 were slightly lower than average while 2017 and 2018 were higher than normal with 2018 having higher SWE than 2017. The amount of SWE dictates the quantity of runoff in the following year, i.e. 2016 SWE would impact spring runoff and samples taken in the winter of 2017. The geochemical analysis of the thermal environment indicated a fairly homogenous system over the course of the three years, with no clear pattern emerging except for a steady increase in annual snowpack from 2016 to 2018.

\section{Temporal small molecule profiles}

As the geochemical results did not yield likely factors explaining the archaeal decrease in relative abundance seen in the 16S rRNA data, our next step was to characterize microbial intracellular small molecules using LC-MS. This analysis of intracellular small molecules extracted from cell pellets suggested that a metabolic transition had occurred. A 2D-PCA score plot of the global small molecule metabolic profiles for each year indicated a contrast in metabolic activity between 2017 and both 2018 and 2019 (Figure 5A). Intracellular small molecules from 2018 and 2019 were somewhat different, but were more closely related than those from 2017. Specific identified MS features that most discriminate the groups using an ANOVA include hypotaurine and butyric acid (Supplemental Figure 1A).

Supplemental Figure $1 \mathrm{~A}$ also indicates that springs cluster by year using the top 10 discriminating intracellular features.

Extracellular small molecules extracted from the sediment exhibited a similar trend wherein 2017 is markedly different than 2018 and 2019 (Figure 5B). The variation between years was most pronounced in the 2017 FS1 and FS5 sediment environments. Exploring the small molecule profiles that most 
differentiate the springs and years via a heatmap indicated the abundance of specific sulfur and nitrogen containing species in spring sediment from 2017 (Supplemental Figure 1B). A general trend indicating a decrease in the number of nitrogen, sulfur, and combined nitrogen and sulfur containing compounds was observed in the thermal sediments each year of the study (Figure 5C). Nitrogen containing compounds exhibited a 15\% decrease between 2017 and 2019, while sulfur containing compounds exhibited a 30\% decrease over the same time period. To validate the molecular formula assignments and the presence of nitrogen and sulfur, MS features from 50 small molecules were examined (Supplementary Table 1-2). Each of these small molecule features was selected in the following statistical correlations section and all possible molecular formulas were derived using the $\mathrm{m} / \mathrm{z}$ value and a $15 \mathrm{ppm}$ cutoff [30]. $79 \%$ of the possible formulas for the $\mathrm{m} / \mathrm{z}$ values of the putative sulfur group contained sulfur. Nitrogen was even more consistent, as $89 \%$ of the possible formulas included nitrogen. Although several formulas were possible for many of the $\mathrm{m} / \mathrm{z}$ mass values measured by LCMS, the presence of nitrogen or sulfur was consistent in the majority of the possible formula assignments. The mass spectrometry-generated small molecules data held a wealth of information, from which emerged a consistent trend where the number of extracellular nitrogen and sulfur containing small molecules decreased each year in the FS system.

\section{$\underline{\text { Statistical correlations }}$}

Correlograms were built to establish whether statistically important variables within the 16S rRNA data correlated with variables from the three corresponding temporal datasets. These correlograms were used to provide a rationale as to the possible mechanisms of archaeal decline in the FS springs over time. Correlograms identify variables that discriminate groups, in this case by year, and then calculate the strength and statistical significance of the correlation. The top 10 most discriminating variables between year and springs were selected from the small molecule datasets and correlated with 16S rRNA sequencing information (Supplemental Figures $2 A-C$ ). With respect to the correlogram with geochemistry, seven of 10 ZOTUs exhibited a significant positive correlation with sulfate concentrations (Supplemental Figure 2A). Zinc concentrations were similarly associated with an increase in the top 10 microbial variables, though this was not as ubiquitous as for the case of sulfate. Sodium, arsenic and dissolved oxygen exhibited an opposite trend, with a negative correlation with most of the selected ZOTUs. Small molecule correlograms indicated a strong correlation between specific sulfur and nitrogen containing compounds and Archaea. Both intra- and extracellular correlograms with ZOTU data were associated with nitrogen and sulfur compounds (Figure 6, Supplemental Figures 2B-C).

A specific examination was conducted using ZOTU data from Archaea and only datasets with nitrogen and sulfur containing small molecules to determine potential drivers of the relative loss of Archaea. This analysis revealed strong positive correlations between specific nitrogen and sulfur containing compounds and Archaea (Figure 6). Not only were almost all correlations positive, i.e. decreases in specific small molecules were observed with decreases in archaeal relative abundance, but the majority of the correlations were significant with a p-value $<0.05$. An archaeal phylogenetic tree with the top 25 discriminating ZOTUs between years that had positive correlations to annual concentrations of nitrogen and/or sulfur containing compounds indicated that the ZOTUs with significant correlations belonged to 
two separate clades of Archaea the phyla Aigarchaeota and Crenarchaeota, both in the TACK superphylum (Figure 7) [22]. This analysis indicated a relationship between specific nitrogen and sulfur containing extracellular small molecules and distinct clades of Aigarchaeota and Crenarchaeota.

\section{Discussion}

This study was undertaken to explore thermoalkaline hot springs and the microbial life that inhabits them to better understand the ecology of these unique environment. The described analysis allowed for the most comprehensive view to date of microbial life in the FS spring system. Our data indicate that a system change likely occurred between 2017 and 2018 which significantly impacted the environment and microbial ecology of the FS springs. Samples from 2017 to 2018 displayed coordinated extracellular small molecule, microbial and intracellular small molecule shifts. However, these general trends did not fully explain the patterns seen in Archaea.

Microbial changes observed over the time period of the study were defined by decreases in relative abundance of archaeal microbes in 2018 and then again in 2019, as prominently indicated by a decline in relative abundance of the TACK superphylum. Relative abundance decreases were also associated with reduced alpha diversity. This is in contrast with the bacterial populations which increased in overall relative abundance in 2018 and 2019, and remained at a somewhat consistent alpha diversity level throughout the study period. This phenomenon of differential responses between bacteria and archaea to a shared environmental stimulus has been observed by Pala, et al., where contrasting archaeal and bacterial abundance shifts were the result of differing environmental factors in the same aqueous environment [31].

Microbial metabolic changes were correlated to a combination of environmental factors. However, the geochemical variables with significant differences over the course of the study did not exhibit a characteristic a pattern reflective of the archaeal population data. Our analysis provided evidence that the strongest correlations belong to associations with specific sulfur and nitrogen containing small molecules. These small molecules decreased steadily from 2017 to 2019 and coincided with a general decrease in the relative abundance and diversity of Archaea. Intracellular small molecule profiles also shifted globally between 2017 and 2018, suggesting metabolic change(s) in the microbial communities of the spring system during this time period.

Archaea play key roles in the nitrogen and sulfur cycles, particularly in extreme environments [32]. Our analysis shows a correlation of metabolic function with nitrogen and sulfur containing extracellular compounds. This correlation is contrasting between Archaea and Bacteria. These observations lead to the hypothesis that environmental shifts result in different small molecule profiles with contrasting elemental composition and structure, resulting in thermophiles in the FS system adapting metabolic strategies to environmental changes. Alterations in optimal metabolism would then lead to differential success of specific organisms in the FS system, which could explain the shift in population makeup that 
occurred from 2017 to 2019 as defined by the observation of a decline in the relative abundance of Archaea.

Although a general decline in Archaea was noted, two clades of Archaea exhibited a strong positive correlation to the number of nitrogen and sulfur compounds detected. These Archaea belonged to either Aigarchaeota or Crenarchaeota, both members of the TACK superphylum [33]. The top correlating Archaea did not include any members from the other superphyla DPANN, Euryarchaeota or Asgard [34]. Many members of the TACK clade have shown strong functional roles in nitrogen and sulfur cycles which are supported by our data $[35,36]$. The correlations to nitrogen and sulfur containing compounds coupled with known metabolic traits of the TACK superphylum demonstrate the need to further explore the relationship that may exist between the species of Aigarchaeota and Crenarchaeota in the FS system with respect to nitrogen and sulfur metabolism.

While the decline in nitrogen and sulfur containing extracellular small molecules could be due to a microbial influence, Gonisor, et al. concluded that the chemodiversity seen in nearby Octopus Spring was likely not microbially driven [36]. Their interpretation was based on the low biodiversity of thermoalkaline springs and the high level of unique extracellular small molecules. One potential explanation for the loss of nitrogen and sulfur compounds is the differential annual mixing of ground and surface water in the springs, where runoff, groundwater and snowpack can impact spring geochemistry and extracellular small molecule composition [37,38]. In our study, samples were collected in the winter with direct water runoff long over, such that the environment should be fairly stable at the time of collection. However, the amount of groundwater recharge from the previous year may have a significant impact on resulting spring water composition the following winter. The area around WCD had unusually high snowpack in 2017 and 2018 (impacting 2018 and 2019 data) relative to the previous and following years according to the Water Resources Data System \& State Climate Office of Wyoming (www.wrds.uwyo.edu). On March $1^{\text {st }}, 2017$ and 2018 , the snow water equivalent amounted to $110 \%$ and $124 \%$ of the median average for the time of year in, respectively. Snow water equivalent in 2016 was $88 \%$ of the median, potentially impacting 2017 data. This SWE trend indicating an increased snowpack from 2016 to 2018 correlates with the loss of unique nitrogen and sulfur containing compounds and archaeal decline. During the increased runoffs in the 2017 and 2018 season from high snowpack, the hot spring water composition likely went through a significant change leading to distinct differences in environmental small molecule compositions. Runoff from snowpack has been previously theorized to modulate specific populations of Archaea in hot springs. Campbell et al. observed temporal changes in Sulfolobus islandicus populations within different hot springs in YNP that did not correlate with measured geochemical changes observed in the springs [39]. These changes were hypothesized to arise from runoff or other hydrogeochemical perturbations.

In conclusion, our analysis has characterized some of the complexity and dynamism of thermoalkaline spring ecology. It also revealed how thermophiles, in this case Archaea, can survive in such extreme conditions, yet remain sensitive to small environmental perturbations. The combination of standard geochemical techniques combined with novel mass spectrometry small molecule analysis exposed the 
limitations of solely using elemental composition and standard geochemical measurements, such as dissolved oxygen, to assess changes in complex environmental systems. Mass spectrometry analysis identified specific changes in small molecule composition that correlated with archaeal relative abundance in thermoalkaline systems. Such associations would have been missed by using a purely geochemical analysis. Our more comprehensive study revealed that environmental events occurred between 2017 and 2019 that shifted hot spring compound composition, manifesting in sulfur and nitrogen containing compound transitions, correlating with metabolic changes and a relative decrease in Archaea.

\section{Declarations}

Acknowledgements

The authors would like to thank the National Park Service for graciously allowing us to sample in YNP (permit YELL-2017-SCI-5480 to YELL-2020-SCI-5480). We would also like to thank Jesse Thomas and Ganesh Balasubramanian of the Proteomics, Metabolomics, and Mass Spectrometry Facility at Montana State University for their expertise. Funding for the Proteomics, Metabolomics and Mass Spectrometry Facility used in this publication was made possible in part by the MJ Murdock Charitable Trust and the Office of the Vice President for Research and Economic Development at MSU. We thank the WM Keck Foundation for providing funding for this research.

\section{Funding}

This research was funded by a grant from the WM Keck Foundation. B.S. was supported by NSF REU grant (CHE1852214). Funding for Proteomics, Metabolomics and Mass Spectrometry Facility used in this publication was made possible in part by the MJ Murdock Charitable Trust and the Office of the Vice President for Research and Economic Development at MSU.

\section{Conflicts of Interest}

The authors declare no conflicts of interest.

\section{Availability of Data and Material}

The authors have included all relevant data and material including mass spectrometry and metabarcoding data.

Code availability.

Not applicable

Author's contributions 
JP conceived the study, collected samples for MS analysis, performed the small molecule extraction and analysis, performed the MS analysis, interpreted the MS and compiled analysis data and wrote the manuscript. RM collected samples, performed the $16 \mathrm{~S}$ analysis and assisted with manuscript preparation. DS collected samples, performed the geochemical analysis and assisted with manuscript preparation. MM collected samples and assisted with manuscript preparation. SK collected samples and performed the small molecule extractions. EB performed the small molecule extractions. BS collected samples and performed the small molecule extractions. VC conceived the study and assisted with manuscript preparation. BB conceived the study, interpreted the results and assisted with manuscript preparation. BP conceived the study, collected samples, interpreted the results and assisted with manuscript preparation.

\section{References}

1. MUELLER, R. C., PEACH, J. T., SKORUPA, D. J., COPIÉ, V., BOTHNER, B. \& PEYTON, B. M. 2020. An emerging view of the diversity, ecology, and function of Archaea in alkaline hydrothermal environments. FEMS Microbiology Ecology.

2. LÓPEZ-LÓPEZ, O., CERDÁN, M.-E. \& GONZÁLEZ-SISO, M.-I. 2015. Thermus thermophilus as a Source of Thermostable Lipolytic Enzymes. Microorganisms, 3, 792-808.

3. SAHAY, H., YADAV, A. N., SINGH, A. K., SINGH, S., KAUSHIK, R. \& SAXENA, A. K. 2017. Hot springs of Indian Himalayas: potential sources of microbial diversity and thermostable hydrolytic enzymes. 3 Biotech, 7, 118.

4. PATEL, A. K., SINGHANIA, R. R., SIM, S. J. \& PANDEY, A. 2019. Thermostable cellulases: Current status and perspectives. Bioresour Technol, 279, 385-392.

5. DECASTRO, M.-E., RODRÍGUEZ-BELMONTE, E. \& GONZÁLEZ-SISO, M.-I. 2016. Metagenomics of Thermophiles with a Focus on Discovery of Novel Thermozymes. Frontiers in microbiology, 7, 15211521.

6. VERMA, P., AJAR NATH YADAV, LIVLEEN SHUKLA, ANIL KUMAR SAXENA AND ARCHNA SUMAN 2015. Hydrolytic enzymes production by thermotolerant Bacillus altitudinis IARI-MB-9 and Gulbenkiania mobilis IARI-MB-18 isolated from Manikaran hot springs. International Journal of Advanced Research, 3, 1241-1250.

7. DE LEON, K. B., GERLACH, R., PEYTON, B. M. \& FIELDS, M. W. 2013. Archaeal and bacterial communities in three alkaline hot springs in Heart Lake Geyser Basin, Yellowstone National Park. Frontiers in Microbiology, 4, 10.

8. BROCK, T. D. 1967. Life at High Temperatures. Science, 158, 1012.

9. CHRISTIANSEN, R. L. 2001. The Quaternary and Pliocene Yellowstone Plateau volcanic field of Wyoming, Idaho, and Montana. Professional Paper. - ed.

10. ROWE, J. J., FOURNIER, R. \& MOREY, G. Chemical analysis of thermal waters in Yellowstone National Park, Wyoming, 1960-65. 1973. 
11. FOURNIER, R., THOMPSON, MICHAEL J., HUTCHINSON, RODERICK A. 1992. The geochemistry of hot spring waters at Norris Geyser Basin, Yellowstone National Park. International symposium on waterrock interactions.

12. THIEL, V., WOOD, J. M., OLSEN, M. T., TANK, M., KLATT, C. G., WARD, D. M. \& BRYANT, D. A. 2016. The Dark Side of the Mushroom Spring Microbial Mat: Life in the Shadow of Chlorophototrophs. I. Microbial Diversity Based on 16S rRNA Gene Amplicons and Metagenomic Sequencing. Frontiers in Microbiology, 7.

13. CAPORASO, J. G., LAUBER, C. L., WALTERS, W. A., BERG-LYONS, D., LOZUPONE, C. A., TURNBAUGH, P. J., FIERER, N. \& KNIGHT, R. 2011. Global patterns of 16S rRNA diversity at a depth of millions of sequences per sample. Proceedings of the National Academy of Sciences of the United States of America, 108, 4516-4522.

14. PARADA, A. E., NEEDHAM, D. M. \& FUHRMAN, J. A. 2016. Every base matters: assessing small subunit rRNA primers for marine microbiomes with mock communities, time series and global field samples. Environmental Microbiology, 18, 1403-1414.

15. APPRILL, A., MCNALLY, S., PARSONS, R. \& WEBER, L. 2015. Minor revision to V4 region SSU rRNA $806 \mathrm{R}$ gene primer greatly increases detection of SAR11 bacterioplankton. Aquatic Microbial Ecology, $75,129-137$.

16. EDGAR, R. C. 2010. Search and clustering orders of magnitude faster than BLAST. Bioinformatics, 26, 2460-2461.

17. EDGAR, R. C. 2016. UNOISE2: improved error-correction for Illumina $16 \mathrm{~S}$ and ITS amplicon sequencing. BioRxiv, Preprint.

18. MURALI, A., BHARGAVA, A. \& WRIGHT, E. S. 2018. IDTAXA: a novel approach for accurate taxonomic classification of microbiome sequences. Microbiome, 6, 140.

19. PARKS, D. H., CHUVOCHINA, M., CHAUMEIL, P.-A., RINKE, C., MUSSIG, A. J. \& HUGENHOLTZ, P. 2020. A complete domain-to-species taxonomy for Bacteria and Archaea. Nature Biotechnology, 38, 10791086.

20. KATOH, K. \& STANDLEY, D. M. 2013. MAFFT Multiple Sequence Alignment Software Version 7: Improvements in Performance and Usability. Molecular Biology and Evolution, 30, 772-780.

21. STAMATAKIS, A. 2014. RAXML version 8: a tool for phylogenetic analysis and post-analysis of large phylogenies. Bioinformatics, 30, 1312-3.

22. LETUNIC, I. \& BORK, P. 2021. Interactive Tree Of Life (iTOL) v5: an online tool for phylogenetic tree display and annotation. Nucleic Acids Research.

23. MATSEN, F. A., KODNER, R. B. \& ARMBRUST, E. V. 2010. pplacer: linear time maximum-likelihood and Bayesian phylogenetic placement of sequences onto a fixed reference tree. BMC Bioinformatics, 11, 538.

24. CHAMBERS, M. C., MACLEAN, B., BURKE, R., AMODEI, D., RUDERMAN, D. L., NEUMANN, S., GATTO, L., FISCHER, B., PRATT, B., EGERTSON, J., HOFF, K., KESSNER, D., TASMAN, N., SHULMAN, N., FREWEN, B., BAKER, T. A., BRUSNIAK, M.Y., PAULSE, C., CREASY, D., FLASHNER, L., KANI, K., MOULDING, C., 
SEYMOUR, S. L., NUWAYSIR, L. M., LEFEBVRE, B., KUHLMANN, F., ROARK, J., RAINER, P., DETLEV, S., HEMENWAY, T., HUHMER, A., LANGRIDGE, J., CONNOLLY, B., CHADICK, T., HOLLY, K., ECKELS, J., DEUTSCH, E. W., MORITZ, R. L., KATZ, J. E., AGUS, D. B., MACCOSS, M., TABB, D. L. \& MALLICK, P. 2012. A cross-platform toolkit for mass spectrometry and proteomics. Nature Biotechnology, 30, 918920.

25. PlUSKAL, T., CASTILLO, S., VILLAR-BRIONES, A. \& ORESIC, M. 2010. MZmine 2: modular framework for processing, visualizing, and analyzing mass spectrometry-based molecular profile data. BMC Bioinformatics, 11, 395.

26. CHONG, J., WISHART, D. S. \& XIA, J. 2019. Using MetaboAnalyst 4.0 for Comprehensive and Integrative Metabolomics Data Analysis. Current Protocols in Bioinformatics, 68, e86.

27. HUBER, W., CAREY, V. J., GENTLEMAN, R., ANDERS, S., CARLSON, M., CARVALHO, B. S., BRAVO, H. C., DAVIS, S., GATTO, L., GIRKE, T., GOTTARDO, R., HAHNE, F., HANSEN, K. D., IRIZARRY, R. A., LAWRENCE, M., LOVE, M. I., MACDONALD, J., OBENCHAIN, V., OLEŚ, A. K., PAGÈS, H., REYES, A., SHANNON, P., SMYTH, G. K., TENENBAUM, D., WALDRON, L. \& MORGAN, M. 2015. Orchestrating high-throughput genomic analysis with Bioconductor. Nature Methods, 12, 115-121.

28. ROHART, F., GAUTIER, B., SINGH, A. \& LÊ CAO, K.-A. 2017. mixOmics: An R package for 'omics feature selection and multiple data integration. PLoS computational biology, 13, e1005752-e1005752.

29. CHONG, J., LIU, P., ZHOU, G. \& XIA, J. 2020. Using MicrobiomeAnalyst for comprehensive statistical, functional, and meta-analysis of microbiome data. Nature Protocols, 15, 799-821.

30. PATINY, L. \& BOREL, A. 2013. ChemCalc: A Building Block for Tomorrow's Chemical Infrastructure. Journal of Chemical Information and Modeling, 53, 1223-1228.

31. PALA, C., MOLARI, M., NIZZOLI, D., BARTOLI, M., VIAROLI, P. \& MANINI, E. 2018. Environmental Drivers Controlling Bacterial and Archaeal Abundance in the Sediments of a Mediterranean Lagoon Ecosystem. Current Microbiology, 75, 1147-1155.

32. OFFRE, P., SPANG, A. \& SCHLEPER, C. 2013. Archaea in biogeochemical cycles. Annu Rev Microbiol, $67,437-57$.

33. CASTELLE, C. J. \& BANFIELD, J. F. 2018. Major New Microbial Groups Expand Diversity and Alter our Understanding of the Tree of Life. Cell, 172, 1181-1197.

34. WILLIAMS, T. A., SZÖLLŐSI, G. J., SPANG, A., FOSTER, P. G., HEAPS, S. E., BOUSSAU, B., ETTEMA, T. J. G. \& EMBLEY, T. M. 2017. Integrative modeling of gene and genome evolution roots the archaeal tree of life. Proceedings of the National Academy of Sciences of the United States of America, 114, E4602-E4611.

35. GUY, L. \& ETTEMA, T. J. G. 2011. The archaeal 'TACK' superphylum and the origin of eukaryotes. Trends in Microbiology, 19, 580-587.

36. WANG, Y., WEGENER, G., HOU, J., WANG, F. \& XIAO, X. 2019. Expanding anaerobic alkane metabolism in the domain of Archaea. Nature Microbiology, 4, 595-602.

37. GONSIOR, M., HERTKORN, N., HINMAN, N., DVORSKI, S. E. M., HARIR, M., COOPER, W. J. \& SCHMITTKOPPLIN, P. 2018. Yellowstone Hot Springs are Organic Chemodiversity Hot Spots. Scientific Reports, 
8, 14155.

38. GIBSON, M. L. \& HINMAN, N. W. 2013. Mixing of hydrothermal water and groundwater near hot springs, Yellowstone National Park (USA): hydrology and geochemistry. Hydrogeology Journal, 21, 919-933.

39. CAMPBELL, K. M., KOURIS, A., ENGLAND, W., ANDERSON, R. E., MCCLESKEY, R. B., NORDSTROM, D. K. \& WHITAKER, R. J. 2017. Sulfolobus islandicus meta-populations in Yellowstone National Park hot springs. Environ Microbiol, 19, 2334-2347.

\section{Figures}
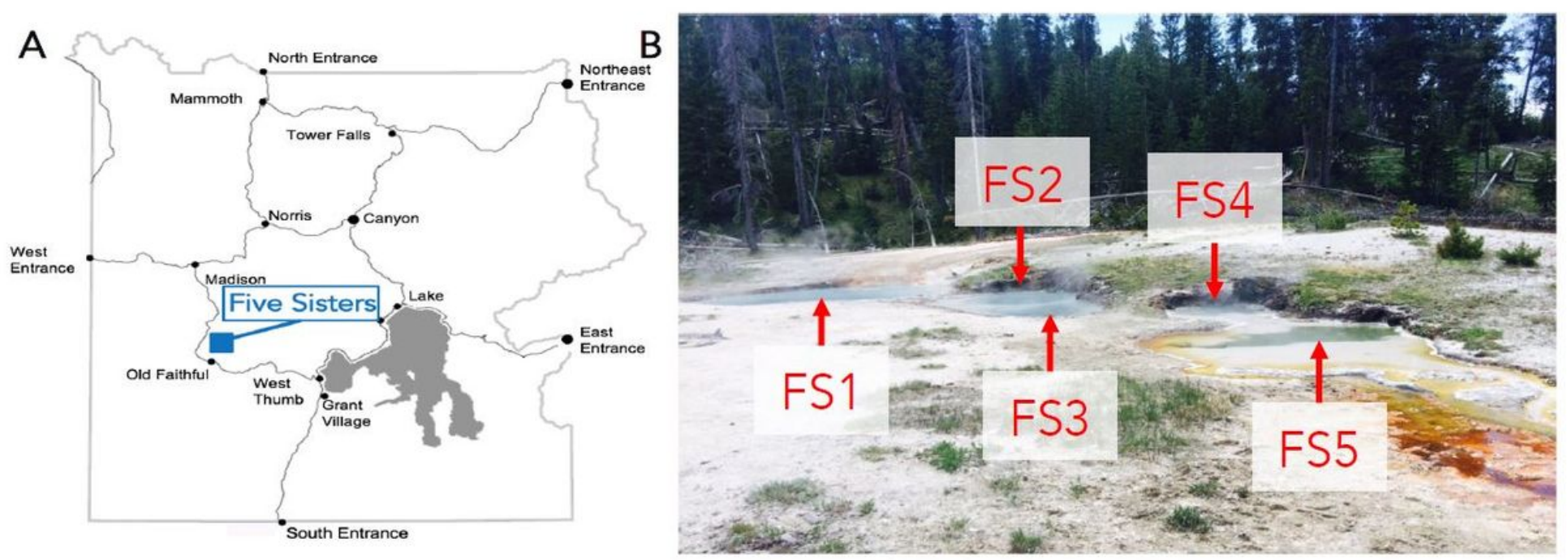

Figure 1

White Creek Drainage and Five Sisters location. (A) Map of YNP indicating the location of the Five Sisters springs north of Old Faithful. (B) Picture of the Five Sisters springs with all five pools labeled. Note: The designations employed and the presentation of the material on this map do not imply the expression of any opinion whatsoever on the part of Research Square concerning the legal status of any country, territory, city or area or of its authorities, or concerning the delimitation of its frontiers or boundaries. This map has been provided by the authors. 
A

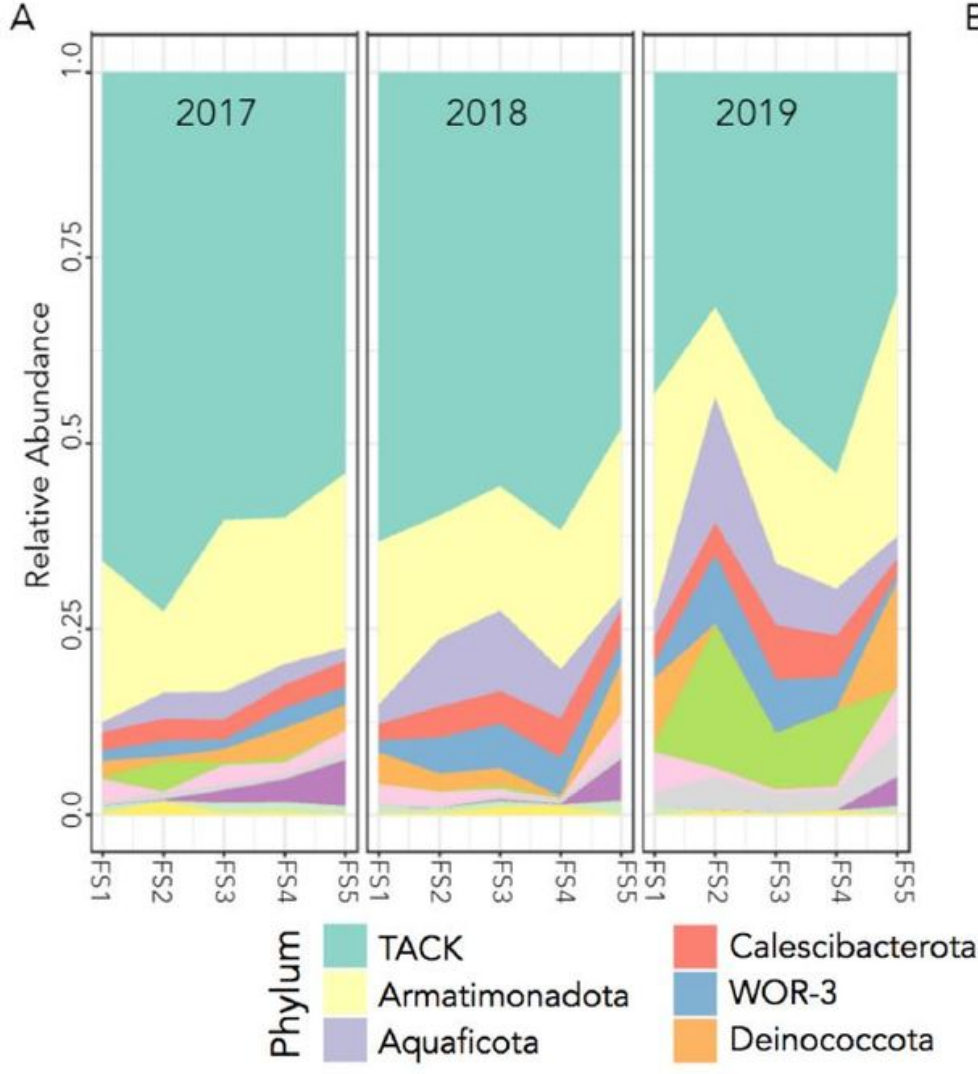

B

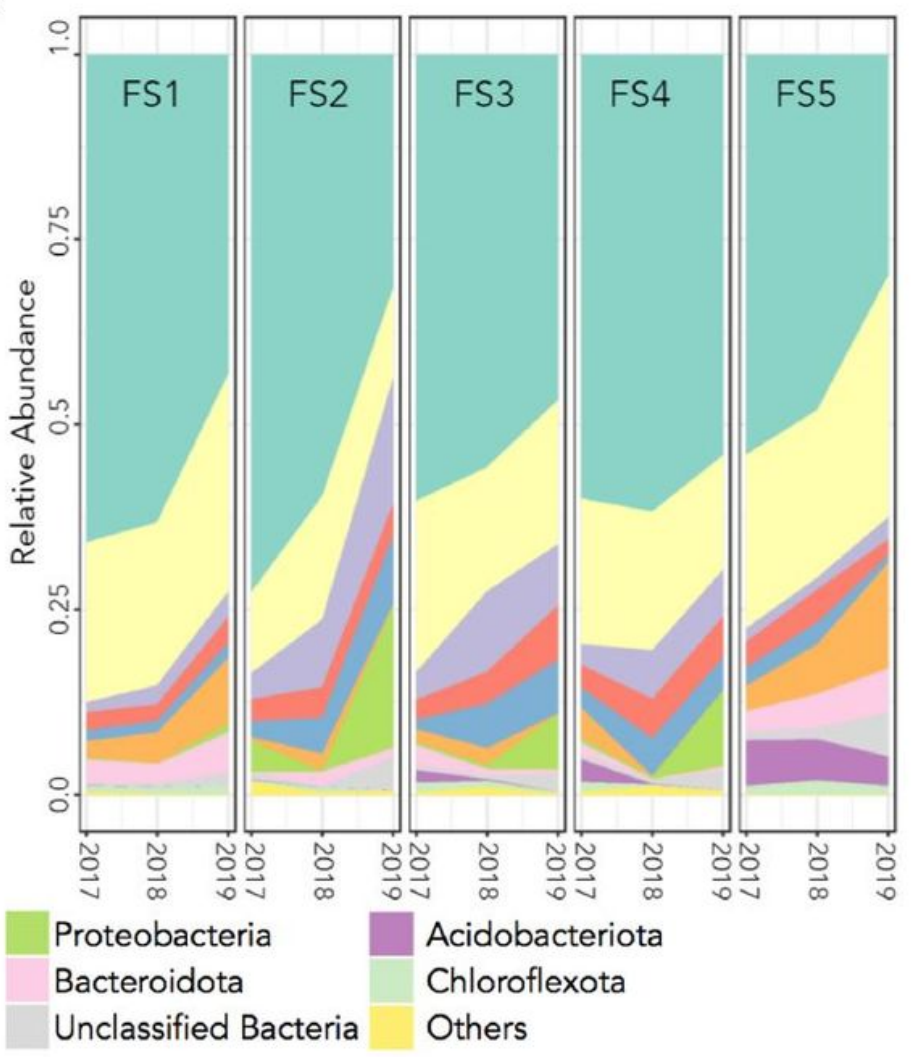

Figure 2

Relative microbial abundance. (A) Phylum-level relative abundance of each spring indicating the microbial population trends for each specific spring over the three-year period. Each panel represents a year from 2017-2019 with springs FS1-FS5 relative abundance levels. (B) Phylum-level abundance for each year in the study. Each panel represents a specific spring over the three-year period. 

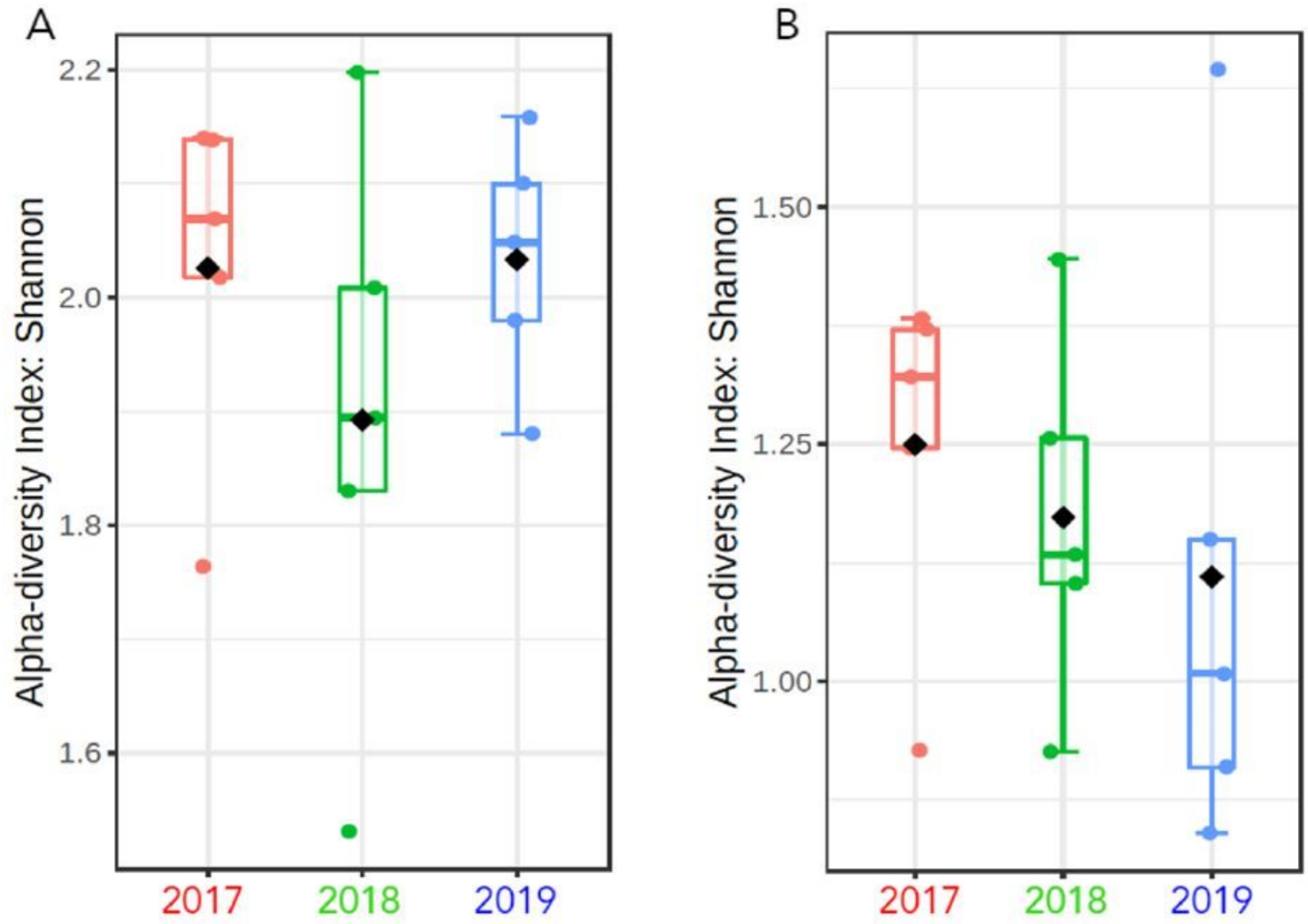

Figure 3

Microbial alpha diversity using a Shannon Index for all springs in a given year. (A) Bacterial alpha diversity from 2017-2019. (B) Archaeal alpha diversity from 2017-2019. Shannon Index analysis accounts for both the evenness and richness in a system. 
A

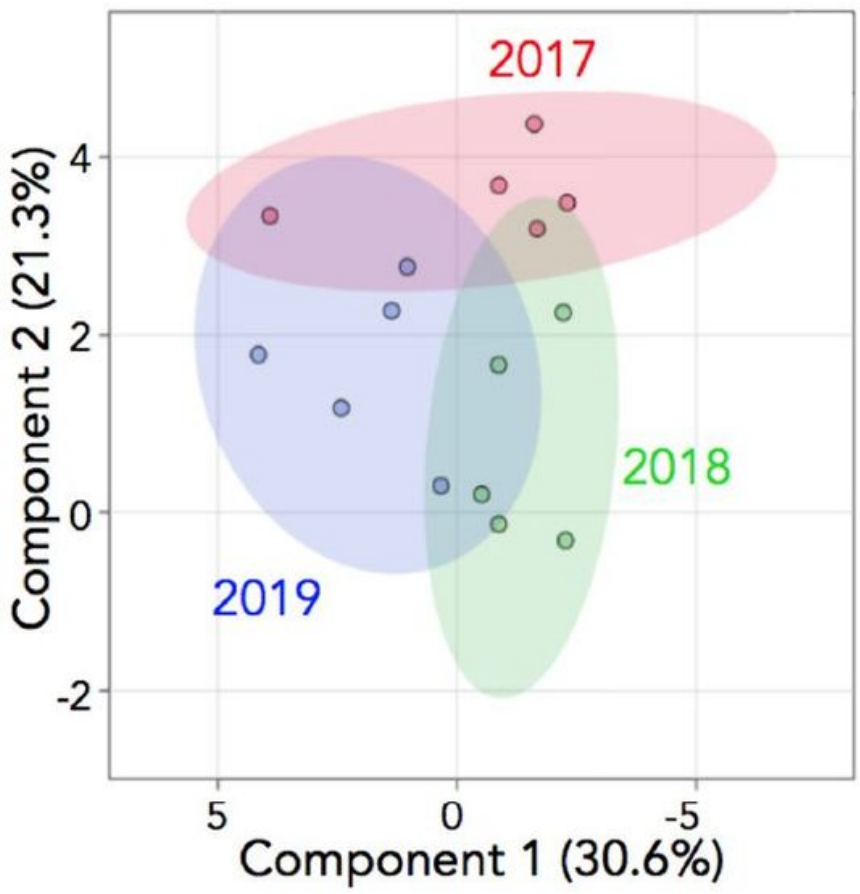

B
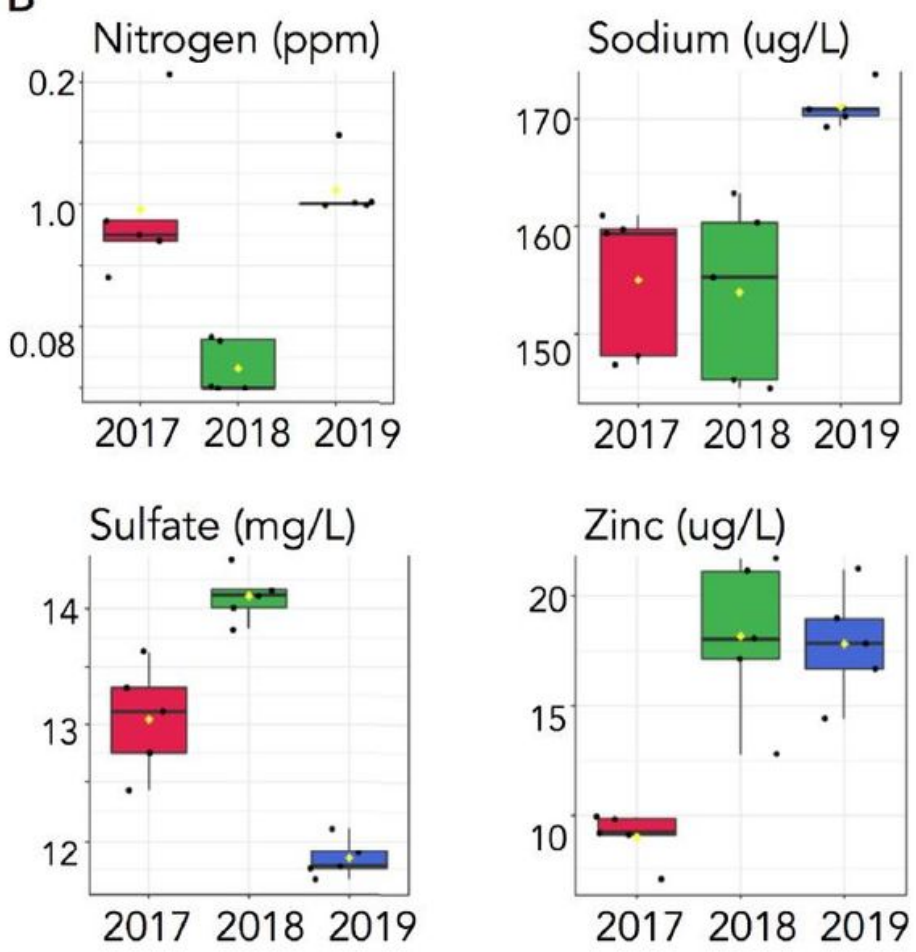

Figure 4

Geochemistry analysis. (A) 2D-PCA scores plot of all five springs by year. Shaded regions indicate $95 \%$ confidense intervals. (B) One-way parametric ANOVA analysis indication geochemical features whose levels are significantly different $(p$-value $<0.05)$ between the three years. 

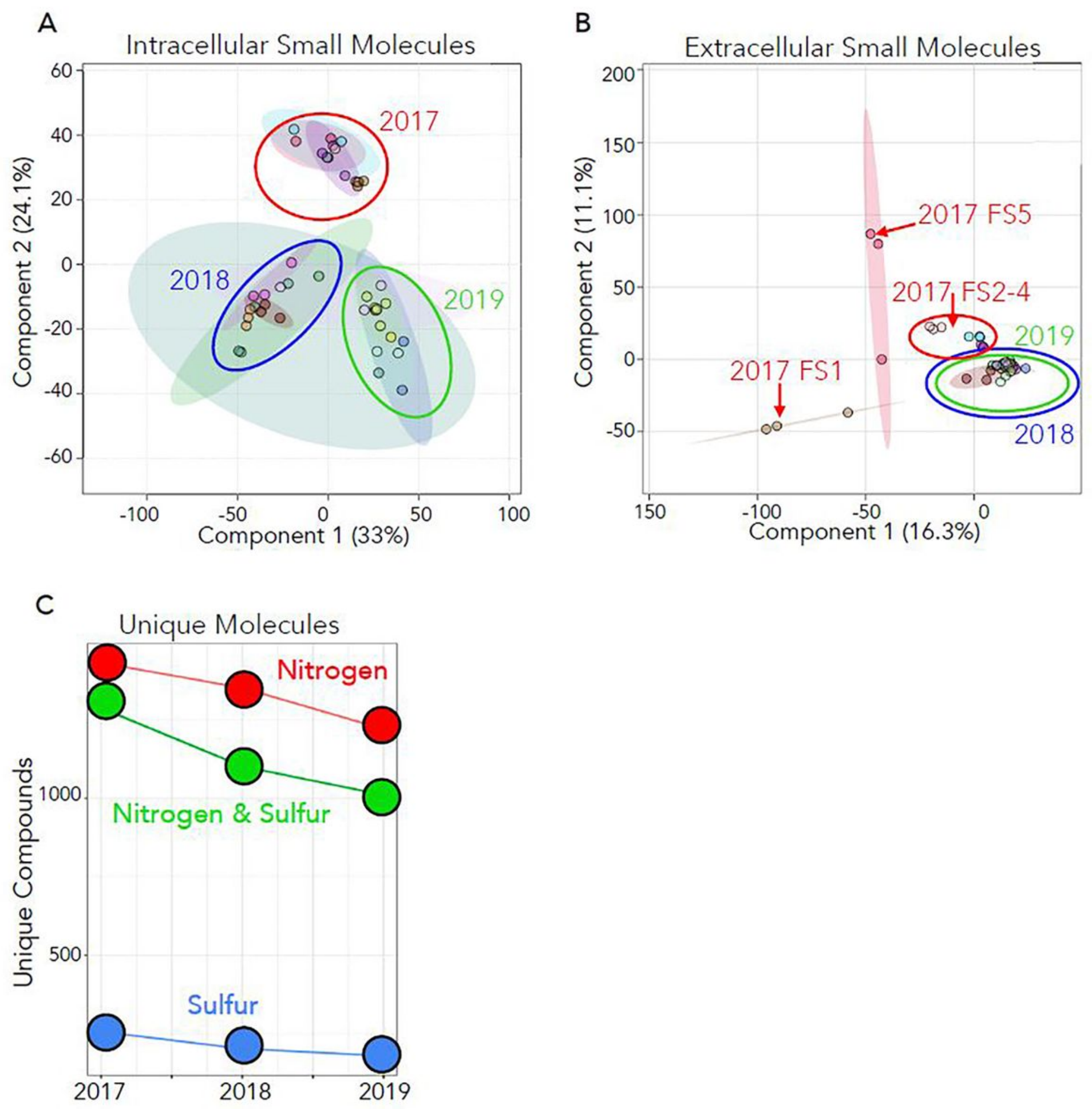

Figure 5

Intracellular and extracellular small molecules analysis. (A) PCA of the intracellular small molecules. Metabolites from 2017 group separately from 2018 and 2019. Metabolites from 2018 and 2019 group independently but overlap in the $95 \%$ confidence intervals for several springs, most strongly in FS4 and FS5. (B) PCA for extracellular small molecules in the sediment. 2018 and 2019 group very closely together relative to 2017. FS2-4 from 2017 group together and are closer to 2018 and 2019 than FS1 and FS5 from 2017. (C) Plot showing unique sulfur, nitrogen and sulfur and nitrogen containing molecules. 
Element specific molecules are shown in each year from 2017 to 2019. Formulas were determined from the mass spectrometry datasets using an error of $15 \mathrm{ppm}$.
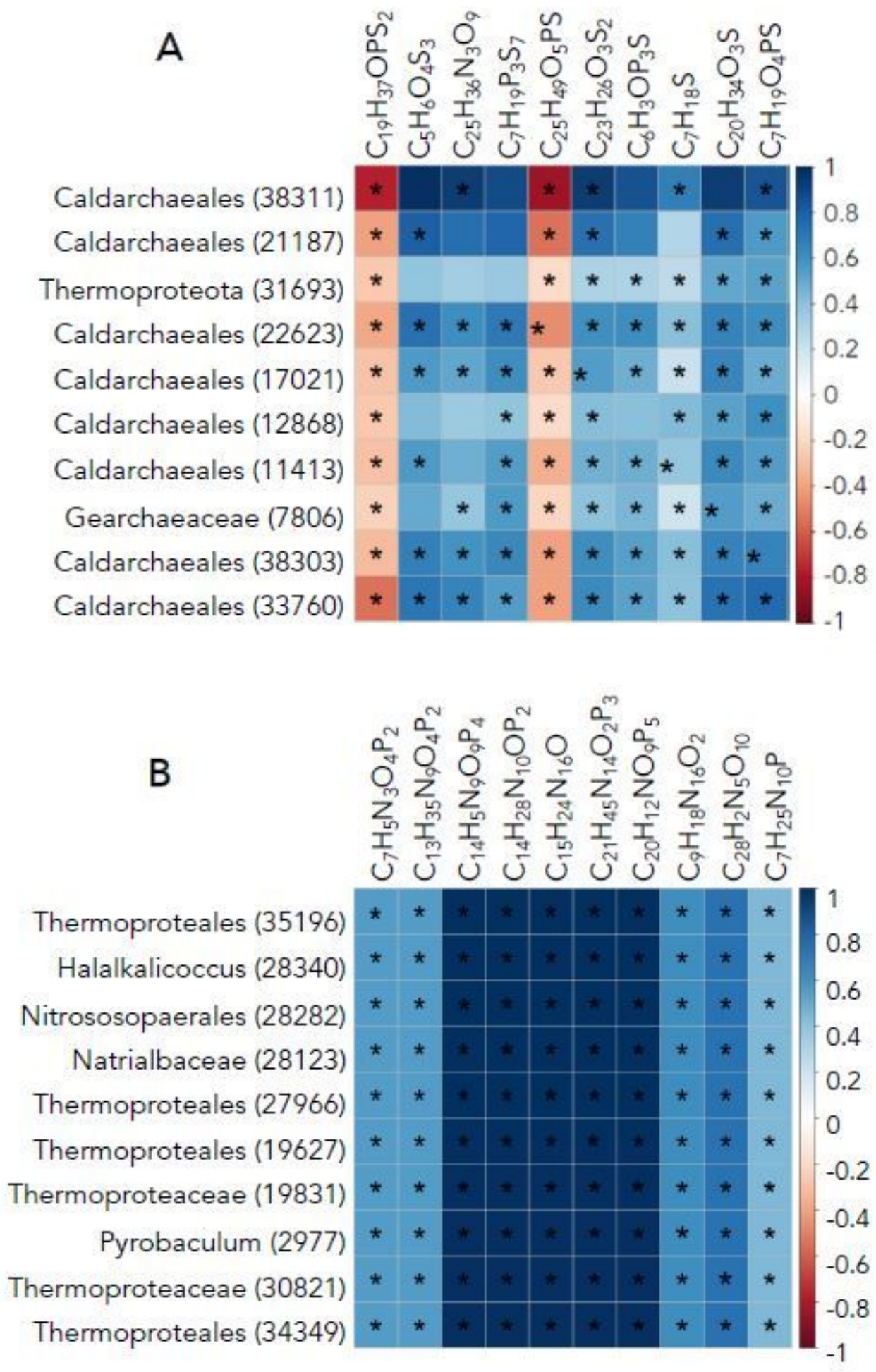

\section{Figure 6}

Correlation analysis. (A) Correlogram as described in the methods section investigating archaeal ZOTUs and sulfur containing extracellular small molecules. (B) Correlogram of archaeal ZOTUs and nitrogen containing extracellular small molecules. The intensity of the color indicates the strength of the 
correlation, with blue demonstrating positive and red demonstrating negative correlations. An asterisk indicates a p-value of $<0.05$ for the correlation.

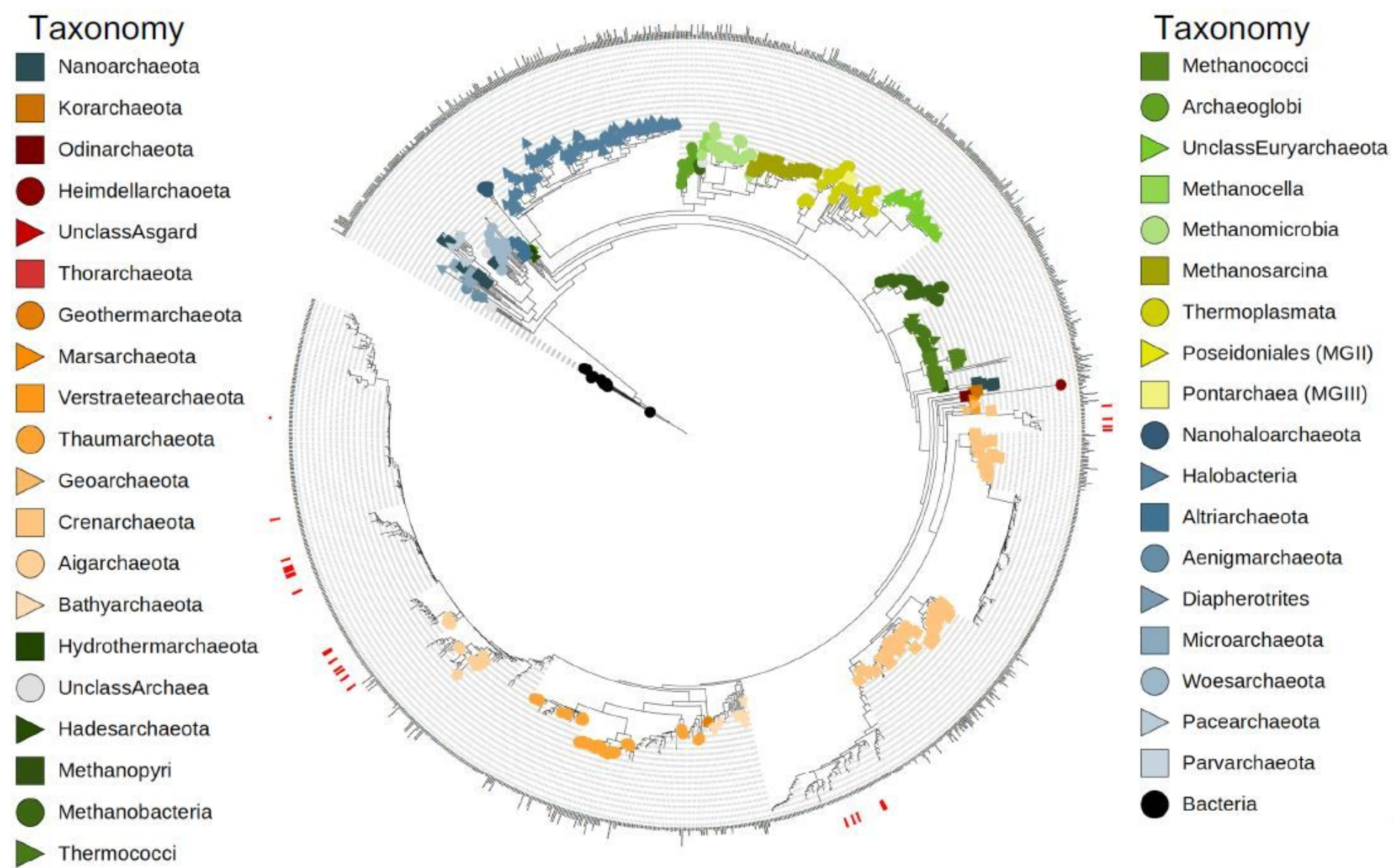

Figure 7

Phylogenetic tree of archaeal sequences. The Inner ring is populated by Archaea found in the Five Sisters site using metagenomic data. The colored shapes in the inner ring indicate phyla of previously cultured and characterized archaeal species corresponding to the taxonomic classifications shown. Archaeal sequences isolated in the correlogram analysis are indicated by red dashes in the outer ring.

\section{Supplementary Files}

This is a list of supplementary files associated with this preprint. Click to download.

- PeachetalSupplemental.pdf 